\title{
Role of magnetic resonance methods in the evaluation of prostate cancer: an Indian perspective
}

\author{
Naranamangalam R. Jagannathan - Virendra Kumar • \\ Rajeev Kumar • Sanjay Thulkar
}

Published online: 20 August 2008

(C) ESMRMB 2008

\section{Erratum to: Magn Reson Mater Phy DOI 10.1007/s10334-008-0122-6}

Figures 2, 3, 4, 5 and 6 were reproduced from the authors' earlier work with permission from the publishers. Unfortunately, however, incorrect reference numbers appeared in the figure legends. The authors regret the error and the inconvenience this may have caused to the readers and are pleased to give the corrected legends here:

Fig. 2 Distribution of ADC values for the PZ (a) and the CG (b) in patients and controls (reprinted from [46] with permission from John Wiley \& Sons Limited)

Fig. 3 Correlation plot of ADC of the PZ and PSA for all groups of patients (reprinted from [46] with permission from John Wiley \& Sons Limited)

Fig. 4 A plot of the metabolite ratio $[\mathrm{Cit} /(\mathrm{Cho}+\mathrm{Cr})]$ versus the ADC value of patients who showed voxels suspicious of malignancy as well as normal portion of the PZ of the prostate (reprinted from [83] with permission from Elsevier) Fig. 5 a T2-weighted sagittal MR image of prostate of a patient (PSA $8.7 \mathrm{ng} / \mathrm{ml}$ ) showing the distance from base of

The online version of the original article can be found under doi:10.1007/s10334-008-0122-6.

N. R. Jagannathan $(\varangle) \cdot$ V. Kumar

Department of NMR, All India Institute of Medical Sciences,

Ansari Nagar, New Delhi 110029, India

e-mail: jagan1954@hotmail.com;nrjgj@yahoo.co.in

R. Kumar

Department of Urology, All India Institute of Medical Sciences,

Ansari Nagar, New Delhi 110029, India

S. Thulkar

Department of Radio-diagnosis, All India Institute of Medical

Sciences, Ansari Nagar, New Delhi 110029, India the prostate of transverse section containing the suspicious voxel. b Voxel grid overlaid over T2-weighted transverse image of the corresponding section showing the distance of the suspicious voxel from midline of prostate. $\mathbf{c}$ MR spectrum obtained from the voxel showing increased Cho and decreased Cit. d TRUS image of MRSI-directed guided biopsy of the prostate of the same patient. The needle mark is the site of biopsy (reprinted from [136] with permission from John Wiley \& Sons Limited)

Fig. $6 \mathrm{~T} 1$ - and T2*-weighted images of a patient whose PSA level is $8.29 \mathrm{ng} / \mathrm{ml}$ recorded $24 \mathrm{~h}$ after biopsy $(\mathbf{a}, \mathbf{b})$ and after 7 days (c, d), respectively. The hemorrhage in T1-weighted image (a) after $24 \mathrm{~h}$ is mainly iso-intense thus seeing the site of biopsy difficult, while the T2*-weighted image (b) showed the site of biopsy clearly (shown by arrows). The hemorrhage on T1-weighted image after a week appears hyper-intense (c) (reprinted from [136] with permission from John Wiley \& Sons Limited) 